\title{
Critical Delays in Clock Distribution Circuits
}

\author{
José R. C. Piqueira and Diego Paolo F. Correa \\ Escola Politécnica da Universidade de São Paulo, Avenida Professor Luciano Gualberto, Travessa 3, \\ No. 158, 05508-900 São Paulo, SP, Brazil \\ Correspondence should be addressed to José R. C. Piqueira; piqueira@lac.usp.br
}

Received 5 August 2013; Revised 28 September 2013; Accepted 13 October 2013

Academic Editor: Jui-Sheng Lin

Copyright ( 2013 J. R. C. Piqueira and D. P. F. Correa. This is an open access article distributed under the Creative Commons Attribution License, which permits unrestricted use, distribution, and reproduction in any medium, provided the original work is properly cited.

\begin{abstract}
As the distribution of clock signals between the nodes of a network became a critical operational requisite, the transmission delays have to be studied because they affect the accurate recovering of the time basis. In this work, the critical delay value is calculated considering simplifications compatible with practical situations. The main contribution is to consider the dissipative terms in the node equations, expressing critical delays, depending on the time constant of the dissipation.
\end{abstract}

\section{Introduction}

The phase control problem in a network of oscillators is a problem that appears in several engineering situations: world wide time signal distribution, synchronous communication networks, distributed computation, processes control, and synchronous operation of micro- and nanocircuits. Generally speaking, the distribution of clock signals can be performed controlling the phase of a node oscillation by using the information of the phases of the other nodes that compose the whole network

Modeling this kind of network presents two types of clock distribution strategies: master-slave (MS) and full-connected (FC), described by Lindsey et al., in a seminal paper that is an important reference for engineers working on design of networks [1].

Concerning the networks conceived up to the end of the last century, Synchronous Digital Hierarchy (SDH), employing MS strategies, was the most important solution becoming a standard recommended by ITU (International Telecommunication Union) [2]. The mathematical analysis of this solution has been widely studied with a lot of different approaches: linear differential equations [3], nonlinear differential equations [4], and stochastic differential equations [5].

Nowadays, it could be considered that clock signal distribution by using MS strategies is well studied and network designers have a lot of good references available for the several types of phase detection and signal filtering [6].
However, the network architecture evolved to complex topologies with phase and frequency references depending, at each node, on the phase and frequency of almost all the other nodes [7] and, consequently, to obtain general solutions becomes feasible only for certain particular situations [8] as the resulting equations for the isolated nodes are secondorder, including a dissipative term. Besides, the coupling terms between the nodes are composed of nonlinear delayed terms [1].

Here, considering that operational frequencies of the node oscillators are normalized, the clock signal phase of each node is supposed to be described by [9]

$$
\ddot{\theta}^{i}(t)+\frac{1}{T^{i}} \dot{\theta}^{i}(t)=K^{i}\left[\sin \left(\phi^{i}(t)-\theta^{i}(t)\right)\right]+f^{i}(t) .
$$

In (1), $T^{i}$ represents a dissipative time constant term, and $K^{i}$ represents a frequency node gain, both related to node $i$. The effect of weak forces is represented by $f^{i}(t)$ that considers noise and small perturbations. A wave propagation term appears as $\phi^{i}(t)$ and it is responsible for considering the dependence of the local phase on the phase of all nodes and is given by

$$
\phi^{i}(t)=\sum_{j=1}^{n}\left(a_{i, j}\right) \theta^{j}\left(t-\tau^{i, j}\right)
$$




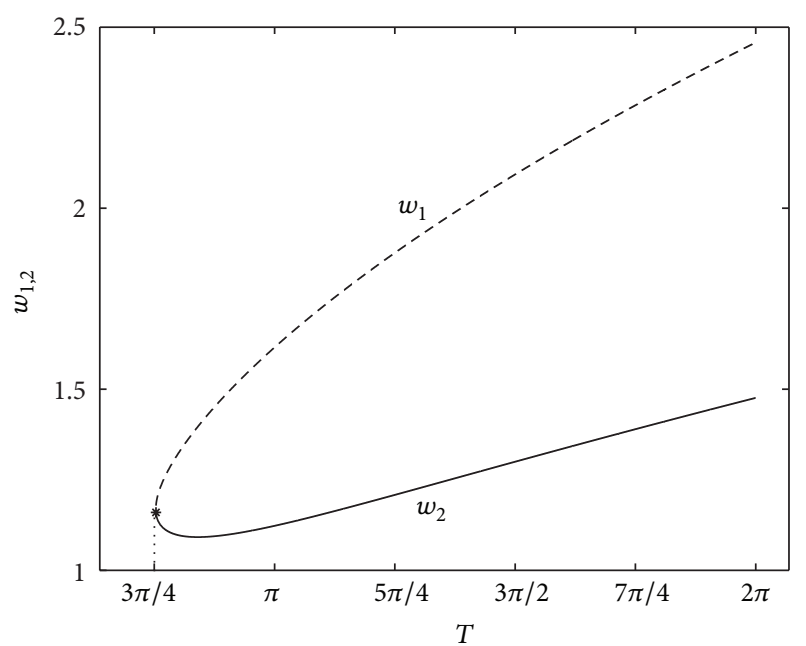

FIGURE 1: Real positive values for $\omega(T)$ given in (19). These values appear for $T>3 \pi / 4$.

In (2), $a_{i, j}$ represents the normalized connection matrix, that is, $\sum_{j=1}^{n}\left(a_{i, j}\right)=1$, for each node, and $\tau^{i, j}$ is the propagation delay between the nodes $i$ and $j$.

The main goal of this paper is to study the several possible behaviors of a network, trying to express them to be related to the connection matrix, the nodes free-running frequencies, and the delays between the nodes. Considering the complexity of the problem, some simplifying assumptions are necessary in order to treat the problem.

The first simplification is about the network structure that is supposed to be triangular [8] as the practical networks are trees, composed of cells that can be modeled as triangles $[1,10]$. They are supposed to have the same parameters. In spite of not being a general case, this assumption provides a simple reasoning with useful practical conclusions, as the circuit components are similar [10].

In the next section, the equations for this type of configuration will be derived with the spatial phase errors being the measurable state variables. Then, in order to analyze the tracking mode of the system, a linear model is developed, resulting in two decoupled equations relating the spatial errors to the delayed spatial errors.

Based on these equations, it is possible to calculate the critical delay that implies instability and to relate it to time constant $T^{i}$ of the dissipative terms. The calculation method for this critical value is presented, followed by numerical results and simulations confirming the conclusions.

\section{Triangular PLL Network}

Following the reasoning and notation exposed in the former section, a three-node PLL network can be modeled by the following equations:

$$
\begin{aligned}
\ddot{\theta}^{(1)}(t)+\frac{1}{T^{(1)}} \dot{\theta}^{(1)}(t) & \\
=K^{(1)} \sin [ & a_{11} \theta^{(1)}(t)+a_{12} \theta^{(2)}\left(t-\tau_{21}\right) \\
& \left.+a_{13} \theta^{(3)}\left(t-\tau_{31}\right)-\theta^{(1)}(t)\right],
\end{aligned}
$$

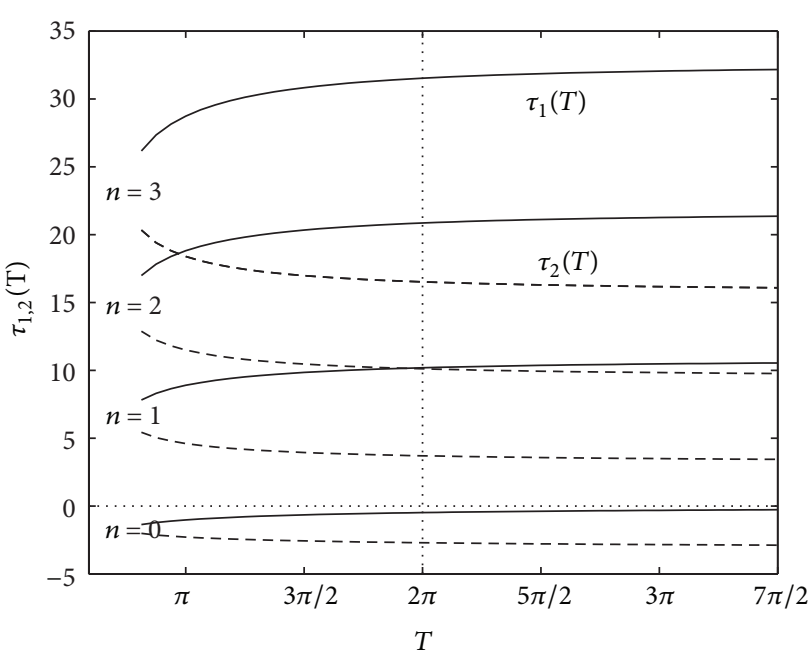

FIGURE 2: $\tau_{c} X T$ following (19) and (20). One curve for each $n$.

$$
\begin{aligned}
& \ddot{\theta}^{(2)}(t)+\frac{1}{T^{(2)}} \dot{\theta}^{(2)}(t) \\
& =K^{(2)} \sin \left[a_{22} \theta^{(2)}(t)+a_{21} \theta^{(1)}\left(t-\tau_{12}\right)\right. \\
& \left.\quad+a_{23} \theta^{(3)}\left(t-\tau_{32}\right)-\theta^{(2)}(t)\right] ; \\
& \ddot{\theta}^{(3)}(t)+\frac{1}{T^{(3)}} \dot{\theta}^{(3)}(t) \\
& =K^{(3)} \sin \left[a_{33} \theta^{(3)}(t)+a_{31} \theta^{(1)}\left(t-\tau_{13}\right)\right. \\
& \left.\quad+a_{32} \theta^{(2)}\left(t-\tau_{23}\right)-\theta^{(3)}(t)\right] .
\end{aligned}
$$

At this point, it is assumed that the nodes have the same constitutive parameters, that is, $T=T^{(1)}=T^{(2)}=T^{(3)}$ and $K=K^{(1)}=K^{(2)}=K^{(3)}$. These assumptions are reasonable considering a practical communication point of view, as the nodes are composed of standard blocks [6]. Besides, due to the same motive, it is considered that all nodes have the same accuracy and, consequently, the phases of the three nodes are used to adjust the phase of the local node with the same weight, resulting that $a_{i, j}=1 / 3, \forall(i, j)[1]$.

In order to be more accurate from an engineering point of view, the delays between the nodes ought to be different. But in real modern networks, they assume low values, being fractions about $10 \%$ of the period of the VCO oscillations, that is, $10 \%$ of the node free-running period. Therefore, here all the delays are considered to be equal, that is, $\tau_{i, j}=\tau, \forall(i \neq j)$, and the results can be related to maximum permitted delays for real networks [2].

Taking the former conditions into consideration, the equations of the phase nodes become

$$
\begin{aligned}
\ddot{\theta}^{(1)} & (t)+\frac{1}{T} \dot{\theta}^{(1)}(t) \\
& =K \sin \left[\frac{1}{3}\left[\theta^{(2)}(t-\tau)+\theta^{(3)}(t-\tau)\right]-\frac{2}{3} \theta^{(1)}(t)\right],
\end{aligned}
$$




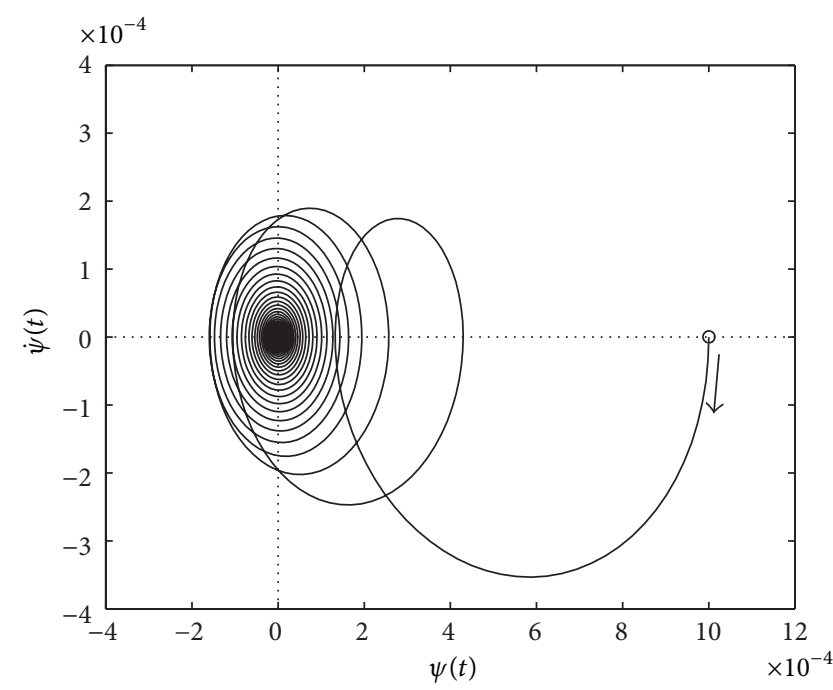

(a)

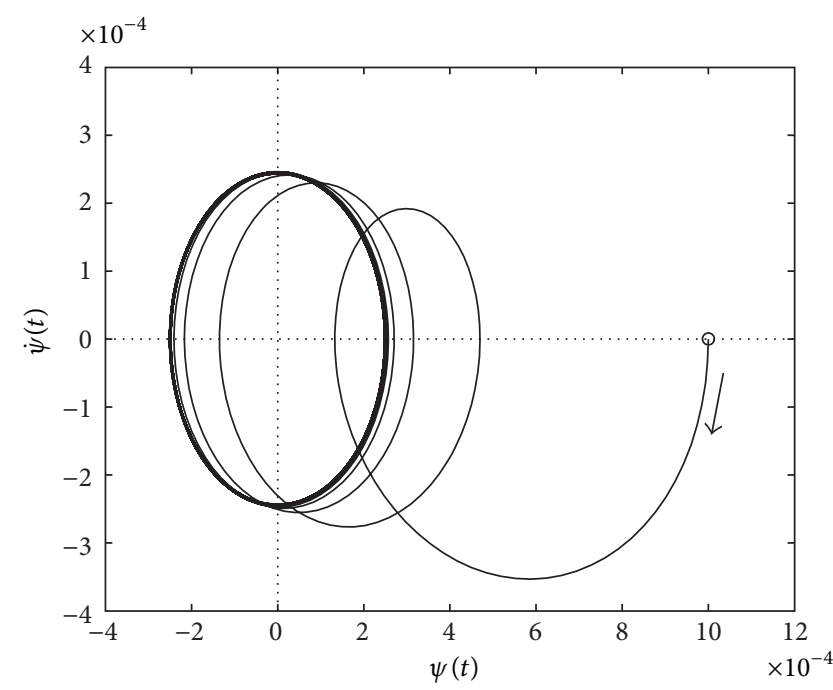

(b)

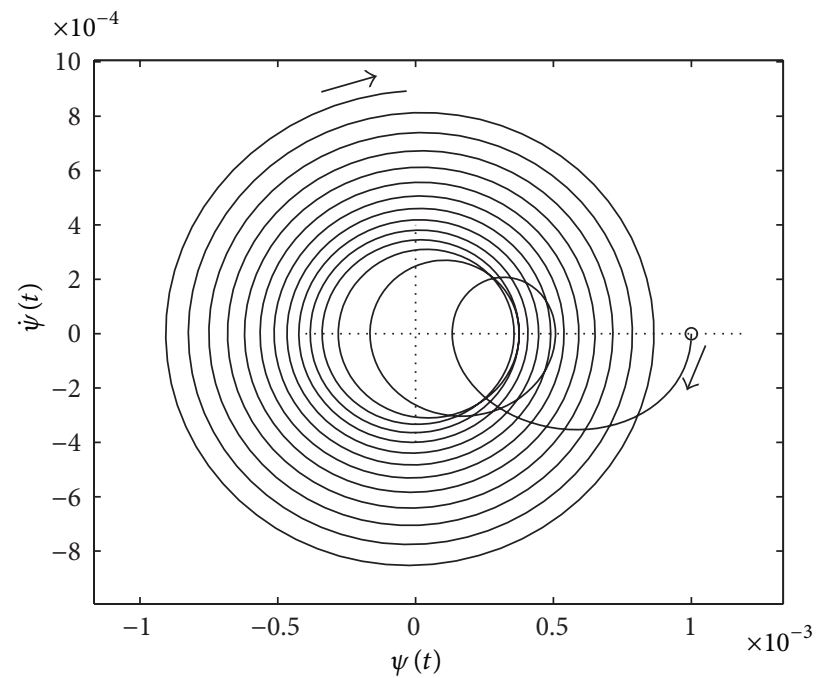

(c)

FIGURE 3: Simulations for (12) with parameter $T=2 \pi$ for three different values of $\tau$. (a) $\tau<\tau_{c}^{1}$; initial condition: $\left(1.0 \times 10^{-1}, 0\right)$; attractor: equilibrium point. (b) $\tau=\tau_{c}^{1}$; initial condition: $\left(1.0 \times 10^{-1}, 0\right)$; attractor: limit-cycle. (c) $\tau>\tau_{c}^{1}$; initial condition: $\left(1.0 \times 10^{-3}, 0\right)$; attractor: infinity.

$$
\begin{aligned}
& \ddot{\theta}^{(2)}(t)+\frac{1}{T} \dot{\theta}^{(2)}(t) \\
& =K \sin \left[\frac{1}{3}\left[\theta^{(1)}(t-\tau)+\theta^{(3)}(t-\tau)\right]-\frac{2}{3} \theta^{(2)}(t)\right] \\
& \ddot{\theta}^{(3)}(t)+\frac{1}{T} \dot{\theta}^{(3)}(t) \\
& =K \sin \left[\frac{1}{3}\left[\theta^{(1)}(t-\tau)+\theta^{(2)}(t-\tau)\right]-\frac{2}{3} \theta^{(3)}(t)\right] .
\end{aligned}
$$

To proceed with the analysis of the network, it must be considered that to obtain the node clocks in a correct sequence, the phase and frequency errors between the nodes have to vanish after any kind of perturbation, with as minimum overshoot and tracking time as possible. Consequently, the important state variables are the spatial phase $\left(\Psi_{i, j}\right)$ and frequency $\left(\dot{\Psi}_{i, j}\right)$ errors, defined as

$$
\begin{aligned}
& \Psi_{i, j}=\theta^{(i)}-\theta^{(j)} \\
& \dot{\Psi}_{i, j}=\dot{\theta}^{(i)}-\dot{\theta}^{(j)} .
\end{aligned}
$$

In this case, a three-node network, there are six index combinations $(i, j)$ defining the spatial errors $\left(\Psi_{i, j}\right)$ and $\left(\dot{\Psi}_{i, j}\right)$. However, if $\left(\Psi_{1,2}\right)$ and $\left(\Psi_{1,3}\right)$, combined with $\left(\dot{\Psi}_{1,2}\right)$ and $\left(\dot{\Psi}_{1,3}\right)$, are chosen, all the other spatial errors can be expressed as 


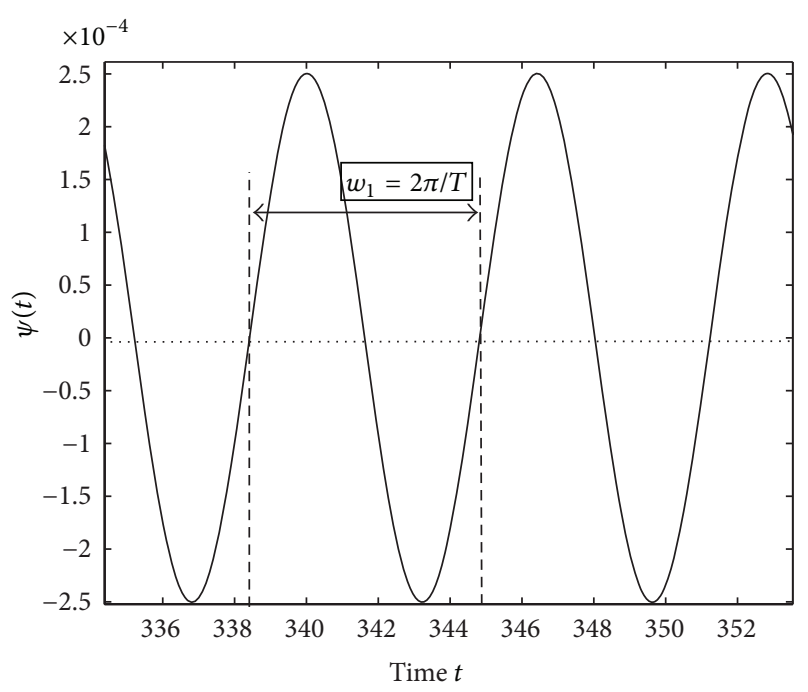

Figure 4: $\psi(t) \times t$ under Figure 3(b) conditions.

linear combinations of them. Therefore, combining (4), the system can be described by

$$
\begin{aligned}
& \ddot{\Psi}_{1,2}+\frac{1}{T} \dot{\Psi}_{1,2} \\
&=K\left\{\sin \left[\frac{1}{3}\left[\theta^{(2)}(t-\tau)+\theta^{(3)}(t-\tau)\right]-\frac{2}{3} \theta^{(1)}(t)\right]\right. \\
&\left.\quad-\sin \left[\frac{1}{3}\left[\theta^{(1)}(t-\tau)+\theta^{(3)}(t-\tau)\right]-\frac{2}{3} \theta^{(2)}(t)\right]\right\},
\end{aligned}
$$

$$
\begin{aligned}
& \ddot{\Psi}_{1,3}+\frac{1}{T} \dot{\Psi}_{1,3} \\
& =K\left\{\sin \left[\frac{1}{3}\left[\theta^{(2)}(t-\tau)+\theta^{(3)}(t-\tau)\right]-\frac{2}{3} \theta^{(1)}(t)\right]\right. \\
& \left.\quad-\sin \left[\frac{1}{3}\left[\theta^{(1)}(t-\tau)+\theta^{(2)}(t-\tau)\right]-\frac{2}{3} \theta^{(3)}(t)\right]\right\} .
\end{aligned}
$$

\section{Analysis of the Tracking Mode}

An important indicator of the performance of a clock distribution systems is regarding its behavior in the tracking mode; that is, how the system recovers the synchronism when a perturbation around the synchronous state occurs [3].

In this case, the phase deviation can be considered to be small and the argument of the sin functions in (7) and (8) can replace the functions [1]. Therefore, the equations describing the system are

$$
\begin{aligned}
& \ddot{\Psi}_{1,2}+\frac{1}{T} \dot{\Psi}_{1,2}+\frac{2}{3} \Psi_{1,2}-\frac{1}{3} \Psi_{1,2}(t-\tau)=0 ; \\
& \ddot{\Psi}_{1,3}+\frac{1}{T} \dot{\Psi}_{1,3}+\frac{2}{3} \Psi_{1,3}-\frac{1}{3} \Psi_{1,3}(t-\tau)=0 .
\end{aligned}
$$

Consequently, the model for the tracking mode of a triangular PLL network is given by two decoupled equations, which are ordinary, homogeneous, and linear, containing a delay term. This equation admits an analytical solution.
According to [11, 12], this type of delay differential equation corresponds to a transcendental characteristic equation, decomposable as

$$
\sum_{j=0}^{N} a_{j} \lambda^{j}+e^{-\lambda \tau} \sum_{j=0}^{M} b_{j} \lambda^{j}=0
$$

which permits to calculate the critical stability value for the delay $\tau$.

\section{Critical Delay Value}

The critical delay value for the three-node clock distribution network can be determined considering that the spatial errors are described by

$$
\ddot{\Psi}(t)+\frac{1}{T} \dot{\Psi}(t)+\frac{2}{3} \Psi-\frac{1}{3} \Psi(t-\tau)=0,
$$

with parameter $T \in \mathfrak{R}^{+}$representing the time constant of the dissipative term and the bifurcation parameter $\tau \in \mathfrak{R}^{+}$ representing the signal processing and propagation delay.

In order to determine how the bifurcation parameter $\tau$ determines the system stability it is necessary, for a given $T$, to know the set $\Gamma \subset \Re^{+}$such that, for a given $\tau=\tau_{c} \in \Gamma$, solutions for (12) change stability.

For the dynamical system described by (12), the characteristic equation is given by

$$
P(\lambda ; T, \tau)=\lambda^{2}+\frac{\lambda}{T}+\frac{2}{3}-\frac{1}{3} e^{-\lambda \tau}=0
$$

and can be written shortly as

$$
P(\lambda ; T, \tau)=a(\lambda ; T)-e^{-\lambda \tau}=0,
$$

where $a(\lambda ; T)=3 \lambda^{2}+(3 / T) \lambda+2$.

It is supposed that there exists $\tau=\tau_{c}$, called critical $\tau$, that leads roots of the characteristic equation up to the imaginary axis, such that $\lambda= \pm j \omega$ with $\left(\omega \in \Re^{+}\right)$, implying the possible onset of a Hopf bifurcation [13].

Consequently, the module condition

$$
|a(j \omega ; T)|=\left|e^{-j \omega \tau_{c}}\right|=1
$$

must hold. Therefore, considering only positive values of $\omega$, from (15), it is possible to find

$$
\omega=\omega(T) .
$$

The number of values for $\omega$ depends on the degree of the polynomial $a(\lambda ; T)$. Considering only the positive and real ones, the roots $\omega$ have to also satisfy the angle condition for (14), given by

$$
\tan ^{-1}\left[\frac{\mathfrak{I}(a(\lambda ; T))}{\mathfrak{R}(a(\lambda ; T))}\right]_{\lambda=j \omega} \pm 2 n \pi=-\omega \tau_{c},
$$

for some $n \in \mathbf{Z}$. 
Then, from (16) and (17), it is possible to find a relationship between a given $T$ and its associated critical $\tau_{c}$ :

$$
\tau_{c}(T)=-\frac{1}{\omega(T)}\left[\tan ^{-1}\left(\frac{\mathfrak{I}(a(\lambda ; T))}{\mathfrak{R}(a(\lambda ; T))}\right)_{\lambda=j \omega(T)} \pm 2 n \pi\right] .
$$

By computing the module condition given by (15), for $a(j \omega ; T)$, there are four possible roots:

$$
\omega(T)= \pm\left[\frac{1}{6 T^{2}}\left(4 T^{2}-3 \pm \sqrt{4 T^{4}-24 T^{2}+9}\right)\right]^{1 / 2},
$$

and replacing (16) into (18) results in

$$
\tau_{c}(T)=-\frac{1}{\omega(T)}\left[\tan ^{-1}\left(\frac{3 \omega(T)}{T\left(2-3 \omega(T)^{2}\right)}\right) \pm 2 n \pi\right] .
$$

Equation (20) shows that, for a given $T$, there are infinite periodical critical values for $\tau$, with period:

$$
\frac{2 n \pi}{\omega(T)}
$$

It can be noticed that there are some situations with (19) having no positive roots, meaning that, for some values of $T$, there is no $\tau_{c}$ that leads the roots up to the imaginary axis. In this case, system stability will depend on the position of the roots for $\tau=0$, for the given $T$.

\section{Numerical Results and Critical Delays}

For a system given by (12), it is possible, by using (19), to calculate which values of $T$ allow critical time delays $\tau_{c}$, responsible for bifurcations characterized by changing stability.

Figure 1 shows the real positive values of $\omega$, given by (19). It can be seen that these roots suddenly appear for values of $T$ greater than $3 \pi / 4$. Analyzing characteristic equation (13), for $\tau=0$, it can be seen that the system described by (12) is stable for every $T \in \mathfrak{R}^{+}$. Then, for $0<T<3 \pi / 4$, the system described by (12) remains stable for every value of time delay.

However, considering a practical point of view, it is important to know how the critical delay values $\left(\tau_{c}\right)$ are related to the dissipative time constant $(T)$. Equations (19) and (20) show that, for a set of values of $T$, there exist families of possible $\tau_{c}$, depending on $n$ (whenever $T>3 \pi / 4$ ), corresponding in (19) to two real positive roots, for each given $T$, each one associated with a critical $\tau_{c}$, as shown in Figure 2.

\section{Engineering Results}

The results presented here enable the design of a clock distribution network with second-order dissipative nodes [1] considering delays between nodes as a complement of the designing technics derived in [7] for delay-free networks.

The general idea is to maintain the delays of the network smaller than the critical value derived here. Under this condition, the reachability of the synchronous state follows the criteria shown in [7].
As the equations considered here are for normalized angular frequency and the time constant $T$ represents the node filter, it must be equal to $2 \pi$, in order to avoid doublefrequency jitter $[14,15]$.

Consequently, taking $T=2 \pi$ and using (19) result in $\omega_{1}=0.9804 \mathrm{rad} / \mathrm{seg}$ and $\omega_{2}=0.5889 \mathrm{rad} / \mathrm{seg}$. From (20), the corresponding critical delay values are $\tau_{c}^{1}=3.7013$ and $\tau_{c}^{2}=10.1853$, for $n=1$.

Simulations of (12) were performed by using DDE23 Matlab's subroutine, with integration step $\Delta t=0.1$ and simulation time $t=1000 \mathrm{~s}$, for three different situations: Figure 3(a): $\tau<\tau_{c}^{1}$, Figure 3(b): $\tau=\tau_{c}^{1}$, and Figure 3(c): $\tau>\tau_{c}^{1}$.

Finally, it can be concluded that $\tau_{c}^{1}$ is a bifurcation parameter. Figure 4 shows a periodic solution, related to Figure 3(b). The angular frequency experimentally measured for $\psi(t)$ is $\omega_{1}=0.98 \mathrm{rad} / \mathrm{seg}$.

\section{Conflict of Interests}

The authors declare that there is no conflict of interests regarding the publication of this paper.

\section{References}

[1] W. C. Lindsey, F. Ghazvinian, W. C. Hagmann, and K. Dessouky, "Network synchronization," Proceedings of the IEEE, vol. 73, no. 10, pp. 1445-1467, 1985.

[2] Timing Requirements of Slave Clocks Suitable for Use as Node Clocks in Synchronization Networks-Recomendation G.812, ITU-T, 1997.

[3] H. Meyr and G. Ascheid, Synchronization in Digital Communications Phase-Frequency-Locked Loops, and Amplitude Control, vol. 1, John Wiley \& Sons, New York, NY, USA, 1990.

[4] Á. M. Bueno, A. A. Ferreira, and J. R. C. Piqueira, "Modeling and filtering double-frequency jitter in one-way masterslave chain networks," IEEE Transactions on Circuits and Systems I, vol. 57, no. 12, pp. 3104-3111, 2010.

[5] A. Mehrotra, "Noise analysis of phase-locked loops", IEEE Transactions on Circuits and Systems I, vol. 49, no. 9, pp. 13091316, 2002.

[6] S. Bregni, Synchronization of Digital Telecommunications Networks, John Wiley \& Sons, New York, NY, USA, 2002.

[7] F. M. Orsatti, R. Carareto, and J. R. C. Piqueira, "Mutually connected phase-locked loop networks: dynamical models and design parameters," IET Circuits, Devices and Systems, vol. 2, no. 6, pp. 495-508, 2008.

[8] J. R. C. Piqueira, M. Q. Oliveira, and L. H. A. Monteiro, "Synchronous state in a fully connected phase-locked loop network," Mathematical Problems in Engineering, vol. 2006, Article ID 52356, 12 pages, 2006.

[9] J. Stensby, "False lock and bifurcation in the phase locked loop," SIAM Journal on Applied Mathematics, vol. 47, no. 6, pp. 1177$1184,1987$.

[10] R. D. Cideciyan and W. C. Lindsey, "Effects of long-term clock instability on master-slave networks," IEEE Transactions on Communications, vol. 35, no. 9, pp. 950-955, 1987.

[11] K. L. Cooke and Z. Grossman, "Discrete delay, distributed delay and stability switches," Journal of Mathematical Analysis and Applications, vol. 86, no. 2, pp. 592-627, 1982. 
[12] J. E. Forde, Delay differential equation models in mathematical biology [Ph.D. thesis], The University of Michigan, 2005, http://www.math.utah.edu/ forde/research/JFthesis.pdf.

[13] J. R. C. Piqueira and L. H. A. Monteiro, "All-pole phase-locked loops: calculating lock-in range by using Evan's root-locus," International Journal of Control, vol. 79, no. 7, pp. 822-829, 2006.

[14] J. R. C. Piqueira and A. Z. Caligares, "Double-frequency jitter in chain master-slave clock distribution networks: comparing topologies," Journal of Communications and Networks, vol. 8, no. 1, pp. 8-12, 2006.

[15] J. R. C. Piqueira and L. H. A. Monteiro, "Considering secondharmonic terms in the operation of the phase detector for second-order phase-locked loop," IEEE Transactions on Circuits and Systems I, vol. 50, no. 6, pp. 805-809, 2003. 


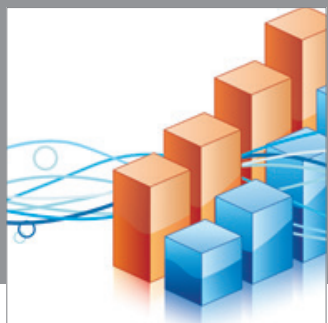

Advances in

Operations Research

mansans

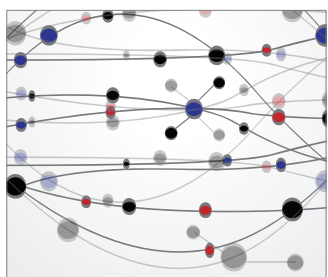

The Scientific World Journal
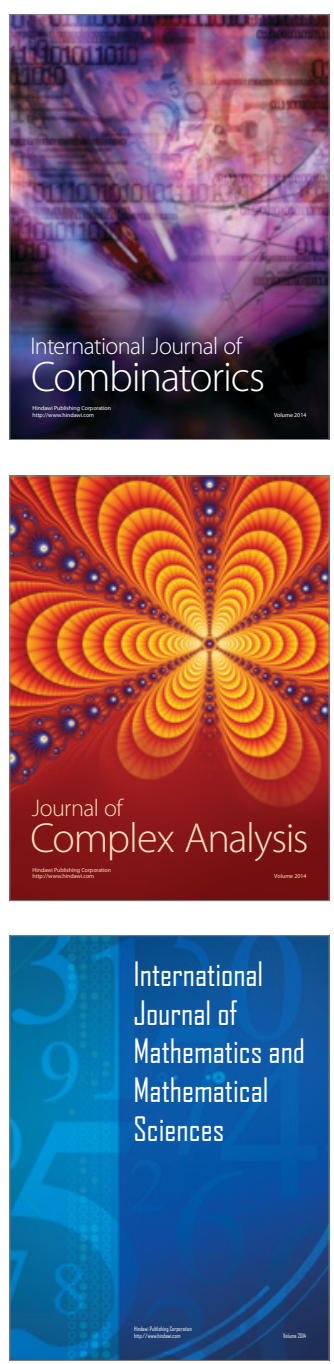
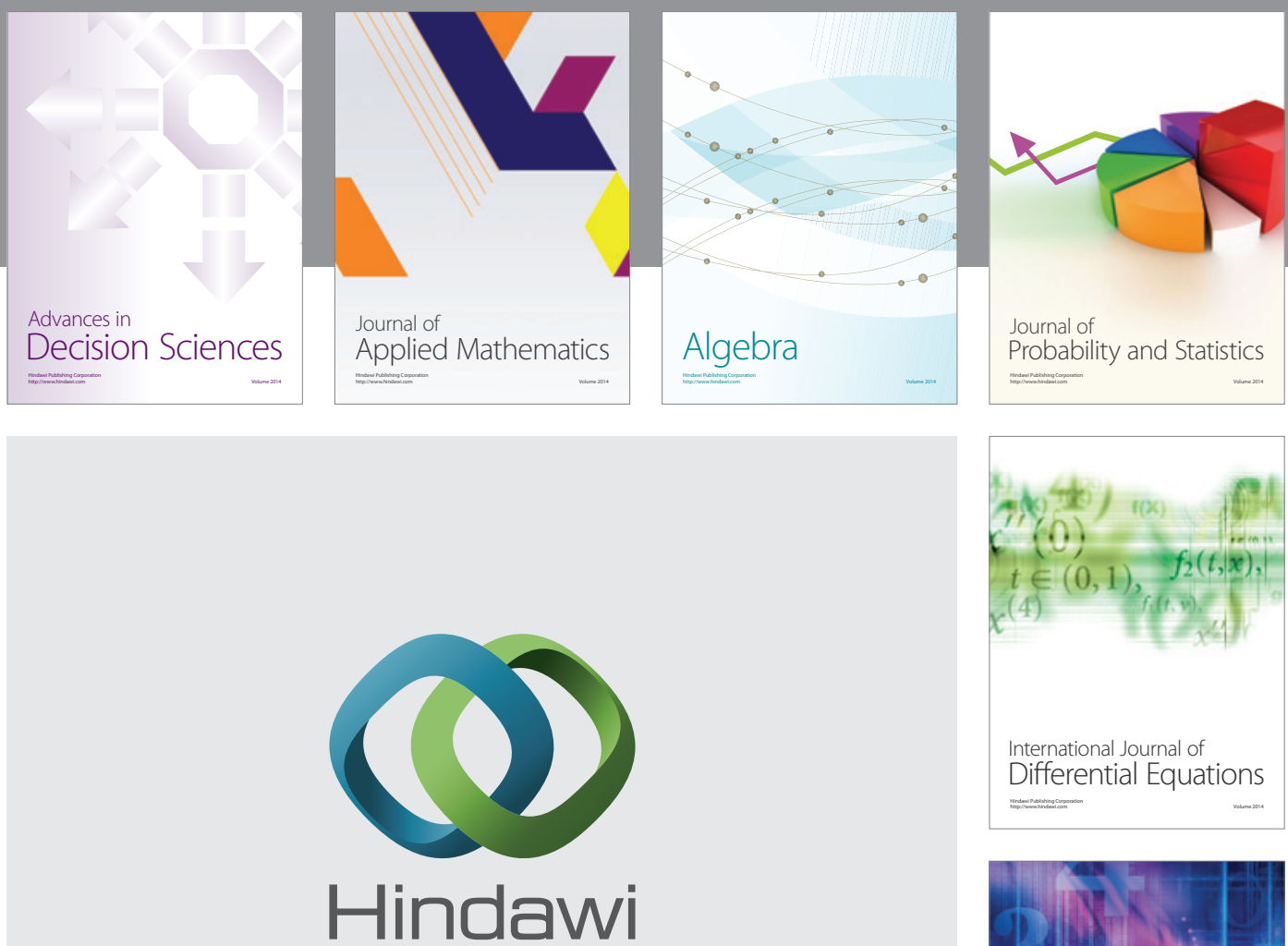

Submit your manuscripts at http://www.hindawi.com
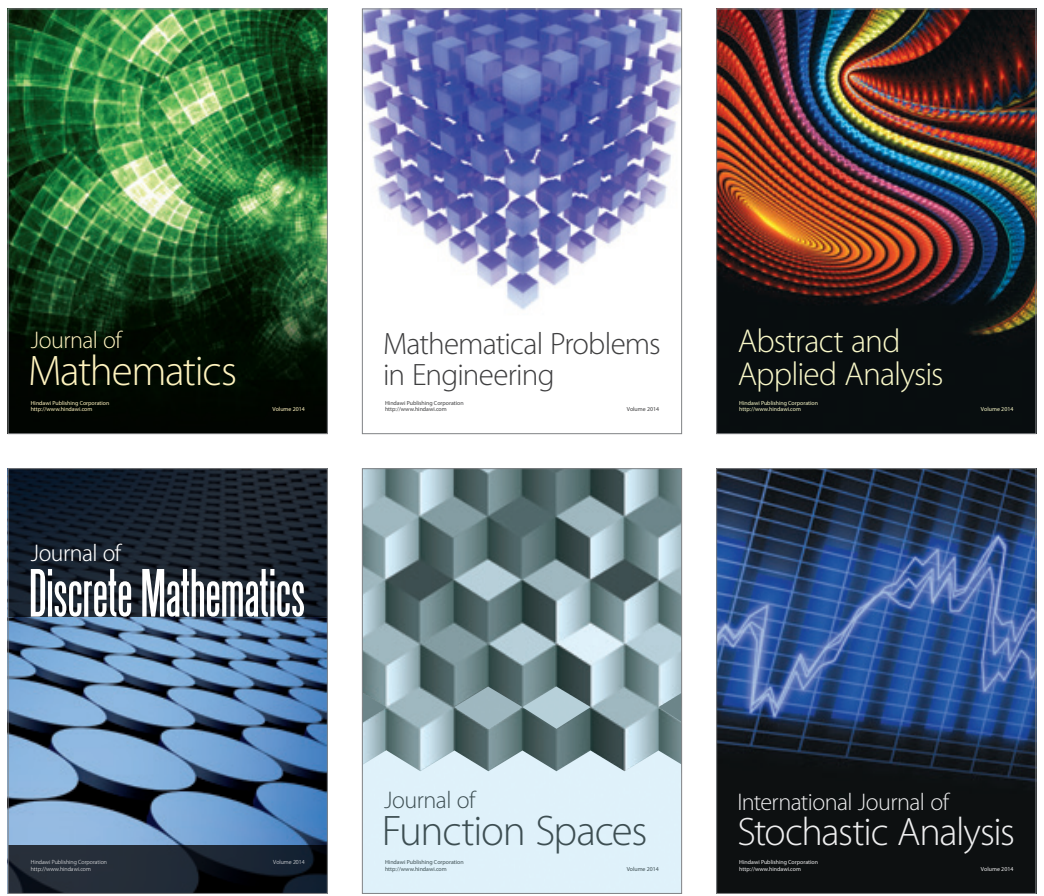

Journal of

Function Spaces

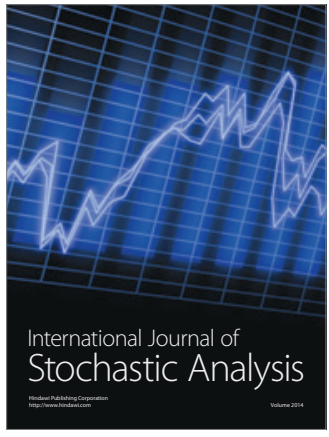

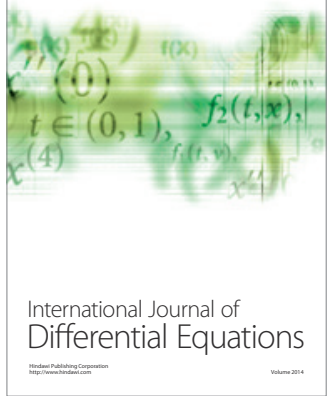
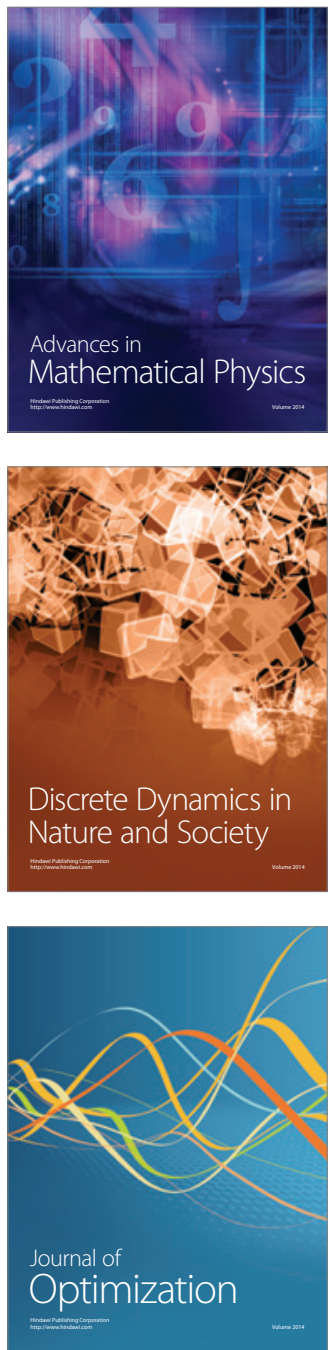